\title{
Michigan State University
}

National Superconducting Cyclotron Laboratory

SOFT DIPOLE RESONANCE IN EXOTIC NUCLEI?

A. GALONSKY, J.J. KRUSE, W.G. LYNCH, D.J. MORRISSEY, N. ORR, J. OTTARSON, D. SACKETT, B.M. SHERRILL, J. WANG, J. WINGER, P. ZECHER, K. IEKI, Y. IWATA, F. DEÁK, Á. HORVÁTH, Á. KISS, Z. SERES, J.J. KOLATA, R.E. WARNER, HUGO SCHELIN, and D.L. HUMPHREY
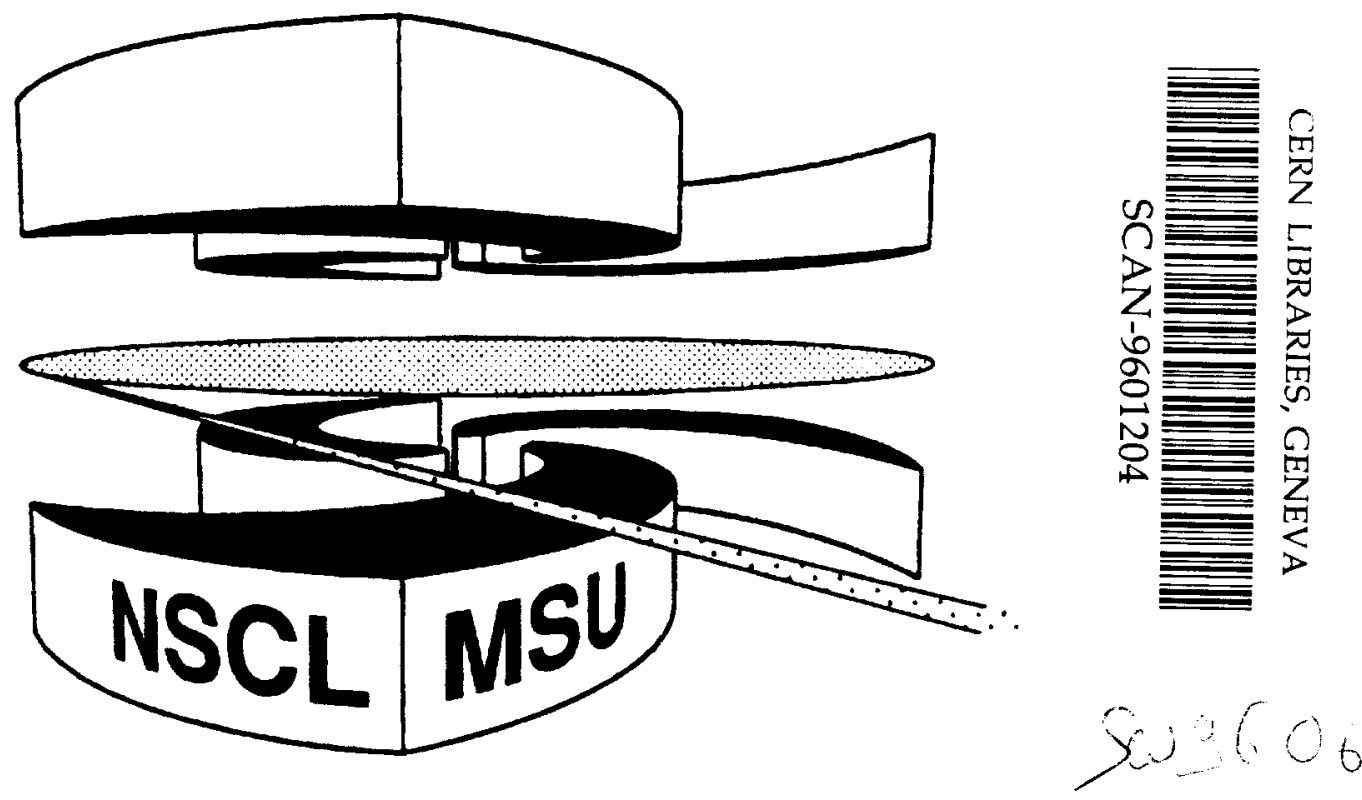

MSUCL-1005

JANUARY 1996 


\section{Soft dipole resonance in exotic nuclei?}

A. Galonsky, J.J. Kruse, W.G. Lynch, D.J. Morrissey, N. Orr*, J. Ottarson, D. Sackett*, B.M. Sherrill, J. Wang, J. Winger ${ }^{\oplus}$ and P. Zecher

Cyclotron Laboratory and Department of Physics \& Astronomy, Michigan State University East Lansing, MI 48824-1321, USA

K. Ieki and Y. Iwata

Department of Physics, Rikkyo University

3-34-1 Toshima, Tokyo 171 Japan

F. Deak, A. Horvath and A. Kiss

Department of Atomic Physics, Eötvös University

Puskin utca 5-7, H-1088, Budapest 8, Hungary

Z. Seres, KFKI, Budapest, Hungary

KFKI Res. Inst. for Particle and Nuclear Physics of the Hungarian Academy of Sciences H-1525 Budapest 114, Hungary

J.J. Kolata

Department of Physics, University of Notre Dame

Notre Dame, IN 46556, U.S.A.

R. E. Warner

Department of Physics, Oberlin College

Oberlin, $\mathrm{OH}$ 44074, U.S.A.

Hugo Schelin

CEFET-Physics Department

80230-901, Curitiba-PR, Brazil

D.L. Humphrey

Department of Physics, W. Kentucky University

Bowling Green, KY 42101 U.S.A.

Projectiles of ${ }^{11} \mathrm{Li}$ were photodisintegrated into ${ }^{9} \mathrm{Li}+\mathrm{n}+\mathrm{n}$ by absorption of a virtual photon in a $\mathrm{Pb}$ target. The momenta of the three particles in the final state were measured, and the dipole strength function was determined up to about $1 \mathrm{MeV}$ above the disintegration threshold. On the average, the ${ }^{9} \mathrm{Li}$ residue had a greater velocity than the neutrons. Interpreted as a post-breakup acceleration of ${ }^{9} \mathrm{Li}$ in the coulomb field of the $\mathrm{Pb}$ nucleus, the lifetime of the excited ${ }^{11} \mathrm{Li}$ was found to be too short for the disintegration process to have gone through a resonant state such as the soft dipole resonance. Because the complete kinematics was measured, we could view the events in the rest frame of ${ }^{11} \mathrm{Li}$. In that frame the distribution of angle between the two neutrons, when the response of the neutron detection system was taken account of, was isotropic. Hence, we have no evidence for a dineutron in "Li. Two recently-constucted neutron walls will be used in future experiments.

Present addresses: * University of Caen; \# Niton Corp., Boston; @ Mississippi State U.

This paper is based on a talk given at the Groningen Conference on Giant Resonances, June 28 - July 1, 1995. 


\section{INTRODUCTION}

Several light nuclei may be called exotic because they are very neutron rich and because they may have special properties when viewed as a core plus two neutrons. Candidate nuclei are ${ }^{6} \mathrm{He}\left(\alpha\right.$-particle core), ${ }^{8} \mathrm{He}\left({ }^{6} \mathrm{He}\right.$ core $){ }^{11} \mathrm{Li}\left({ }^{9} \mathrm{Li}\right.$ core $)$ and ${ }^{14} \mathrm{Be}\left({ }^{12} \mathrm{Be}\right.$ core). In all four nuclei the pairs--core plus neutron and neutron plus neutron--do not bind, but the three-body system is bound. It has been suggested $[1,2]$ that the neutrons in these nuclei form a bound dineutron. "Li has been studied the most. It has the additional property that the two neutrons are so lightly bound that their wavefunctions extend to large distances; they form a diffuse halo around their ${ }^{9} \mathrm{Li}$ core. The halo structure offers the possibility of a collective electric dipole resonance different from the giant dipole resonance. In the so-called soft dipole model [2] "Li may be excited by $\mathrm{E} 1$ photon absorption into a resonance in which the restoring force on the oscillating core is provided by only the two halo neutrons. Hence, the resonant energy should be well below that of the giant dipole resonance, in which all of the neutrons provide the restoring force. Figure 1 gives a pictorial display of these resonances.

\section{Giant Dipole Resonance}
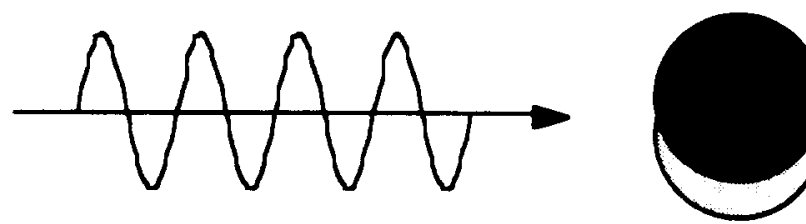

Resonant Energy $\sim 25 \mathrm{MeV}$

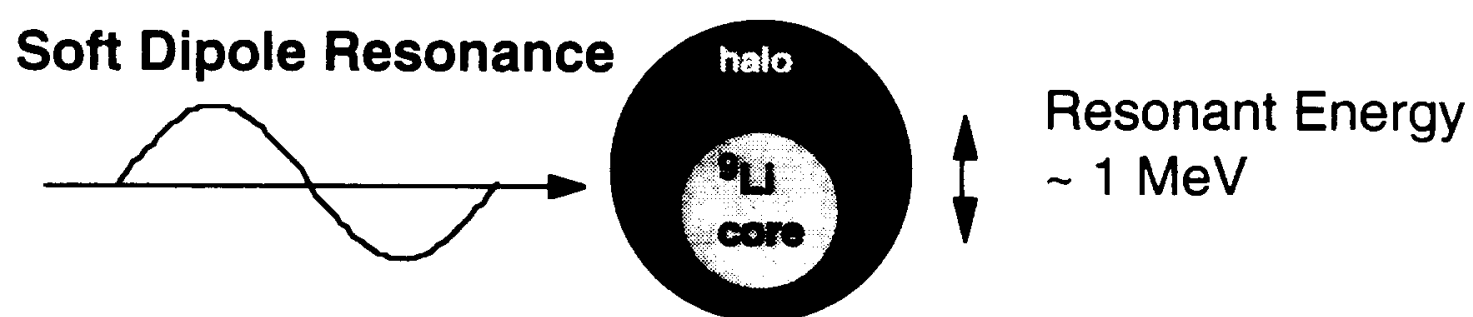

Fig. 1. Pictorial representations of the giant dipole resonance and the soft dipole resonance.

Experiments on the photodisintegration of ${ }^{11} \mathrm{Li}$ were perfomed at MSU [3,4] and at RIKEN [5]. One of the two goals of the MSU experiment was to find the parameter values--resonant energy and width--of the soft dipole resonance.

The other goal was to test the suggestion $[1,2]$ that the two halo neutrons in "Li form a bound dineutron. 
In what follows we will first describe the MSU experiment. Then we will give the results on the soft dipole resonance followed by the results on the dineutron question. Finally, we will discuss some experimental improvements underway at MSU to improve the detection of neutrons in future experiments on dissociation of neutron-rich nuclei.

\section{THE ${ }^{11}$ LI PHOTODISINTEGRATION EXPERIMENT}

The structure of the giant dipole resonance has been studied for more than four decades by measurement of the excitation function for photon absorption. In Fig. 2 are sketches of the energetics for photoneutron disintegration of ${ }^{197} \mathrm{Au}$ and of ${ }^{11} \mathrm{Li}$. The neutron separation energies in the two nuclei are quite different-- $8 \mathrm{MeV}$ for ${ }^{197} \mathrm{Au}$ and $0.3 \mathrm{MeV}$ for ${ }^{11} \mathrm{Li}$. Also, as mentioned in the Introduction, for " $\mathrm{Li}$ the separation energy is for two neutrons, because the core, ${ }^{9} \mathrm{Li}$, and one neutron do not bind. Nevertheless, the photonuclear process is of the same type in both cases. But we cannot do the "Li experiment in the same way as the ${ }^{197} \mathrm{Au}$ experiment. In the ${ }^{197} \mathrm{Au}$ experiment [6] photons

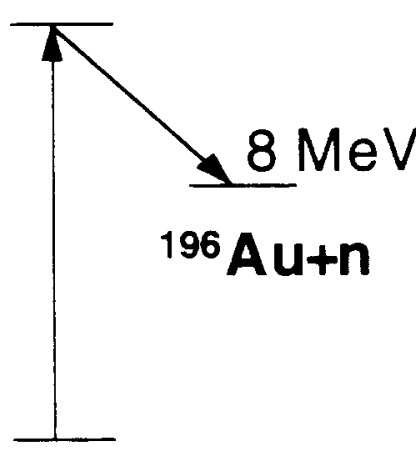

197 AU

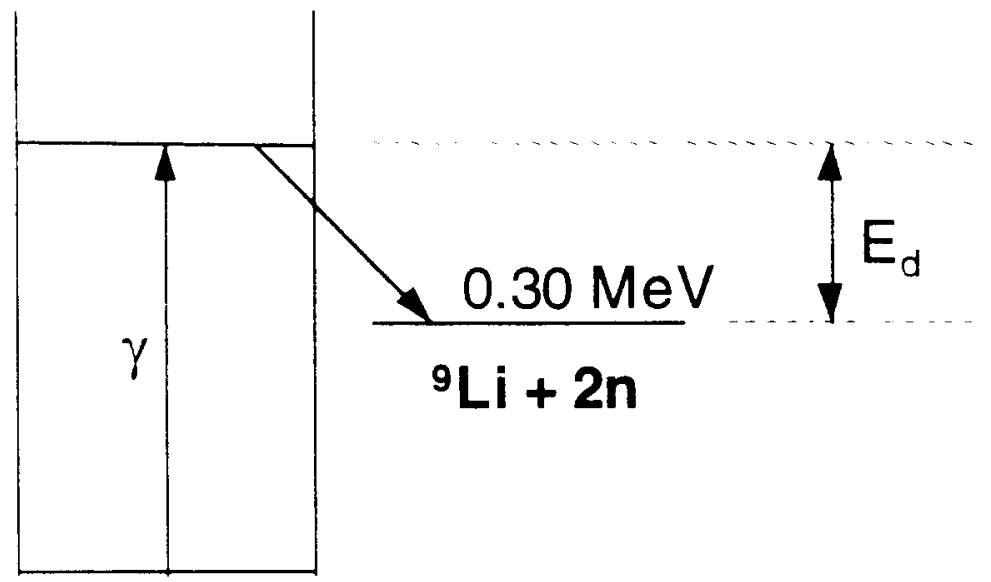

${ }^{11} \mathrm{Li}$

Fig. 2. Energetics of photoneutron disintegration of ${ }^{197} \mathrm{Au}$ and ${ }^{11} \mathrm{Li}$.

of a known energy (varied from 8 to $25 \mathrm{MeV}$ ) were used to bombard a gold target. With the normal meaning of a target, one of ${ }^{11} \mathrm{Li}$ is an impossibility. To photodisintegrate ${ }^{11} \mathrm{Li}$ we therefore reversed the roles of target and projectile, employing two ingenious developments--the radioactive beam facility [7] at Michigan State University and the method of equivalent photons [8.9].

In our experiment $[3,4]^{1}{ }^{1} \mathrm{Li}$ became the projectile, and the electric field of $\mathrm{aPb}$ target nucleus was the photon source. In this procedure the photon spectrum is calculable [9], but we have no control over the energy of the photon absorbed in a given event. E $\gamma$ is 
discovered only after a complete kinematics analysis of the event. Therefore, we had to do a triple-coincidence experiment in which the energies and directions of ${ }^{9} \mathrm{Li}$ and of both neutrons were measured. The kinematic analysis yields the decay energy $E_{d}$, and then

$$
E_{\gamma}=E_{d}+S_{2 n}
$$

where $S_{2 n}$ is the two-neutron separation energy, $0.30 \mathrm{MeV}[10]$ in ${ }^{11} \mathrm{Li}$. The dipole strength function is obtained from the equation

Photon Absorption $\sigma=($ El Strength Function) X (Virtual Photon Spectrum).

A schematic representation of the above procedure is given in Fig. 3. There we see that in the reference frame of the "Li projectile one sees an approaching $\mathrm{Pb}$ nucleus with a Lorentz-contracted electric field. This field can be represented as a virtual photon spectrum $[8,9]$ that is decreasing with photon energy. ${ }^{11} \mathrm{Li}$ is excited when it absorbs one of these photons. Measurement of the decay energy tells us what energy the absorbed photon had. There is a difference between the spectrum of absorbed photons and the spectrum of virtual photons because the $\mathrm{E} 1$ strength function is not a constant; the difference gives us the $\mathrm{El}$ strength function.

\section{$T_{1 / 2}$ of ${ }^{11} \mathrm{Li}=0.01 \mathrm{sec}-->$ target problem}

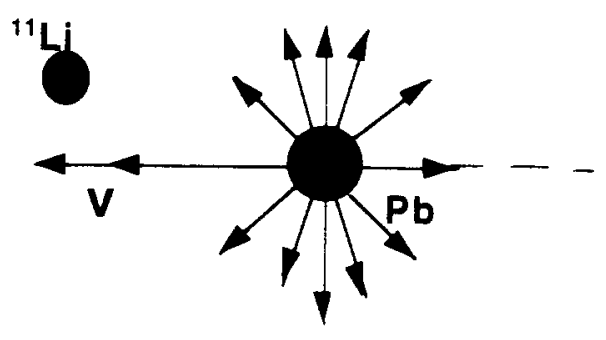

-measurement of $E_{d} \Rightarrow=E_{\gamma}$

- must measure complete kinematics of final state --. 9Li, n, n

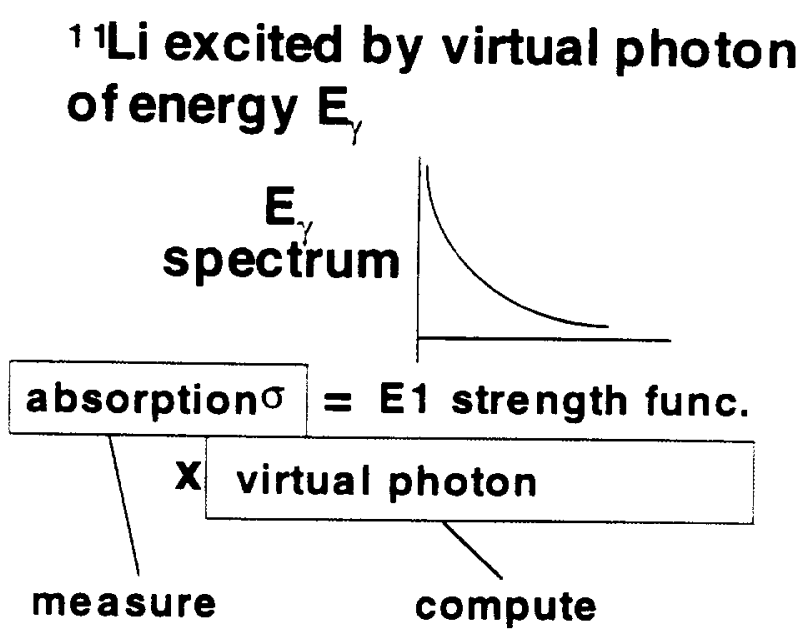

Fig. 3. Steps in the determination of the low-energy $E 1$ strength function for the radioactive nucleus ${ }^{11} \mathrm{Li}$. 
The complete kinematics were measured with the apparatus illustrated in Fig 4. The beam was incident from the lower right at an energy of $30 \mathrm{MeV} /$ nucleon. Each ${ }^{11} \mathrm{Li}$ projectile had already generated a time-of-flight start signal in a scintillator. From its signal

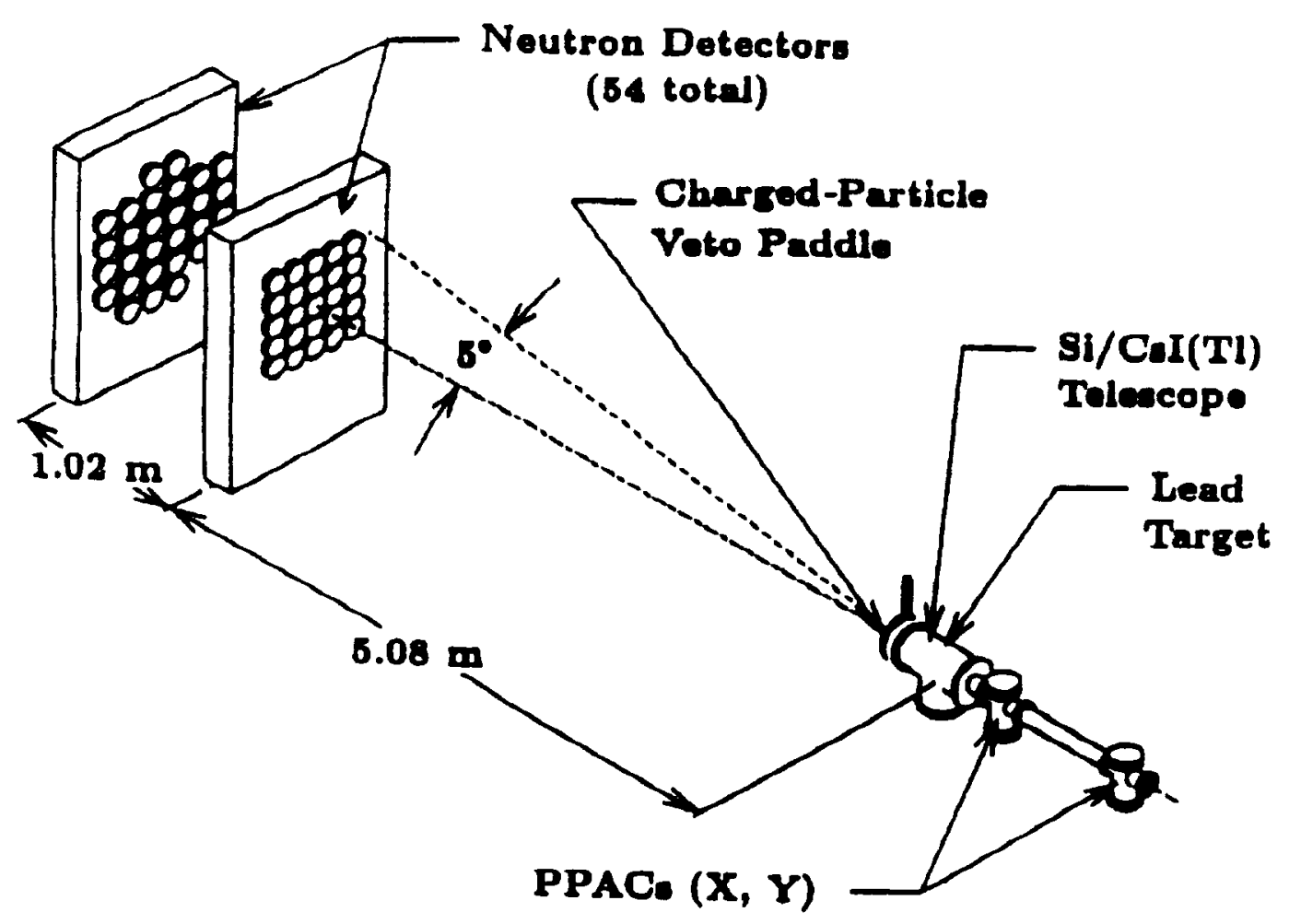

Fig. 4. Layout of the experiment. The 25 neutron detectors in the front layer were $5 \mathrm{~m}$ from the $\mathrm{Pb}$ target, the back layer $6 \mathrm{~m}$.

in the $\Delta \mathrm{E}$ Si detector, we could determine its kinetic energy, and from the two positionsensitive PPAC (x,y) signals, its direction. Energy and direction of the ${ }^{9} \mathrm{Li}$ decay fragment were measured with the Si/CsI telescope; the Si had 16x and 16y strips, effectively defining 256 pixels of angular width $1.2^{\circ}$. Energy and direction of each neutron were determined from flight time and neutron detector (liquid scintillator) position. The maximum detectable neutron angle was only $5^{\circ}$.

\section{RESULTS ON THE SOFT DIPOLE RESONANCE}

\subsection{E1 Strength Function}

The decay energy spectrum is shown in the left half of Fig. 5. Where the statistical accuracy is good, i.e., up to $E_{d}=0.8 \mathrm{MeV}$, the spectrum is about the same as in the RIKEN [5] experiment. In the right half is the deduced electric dipole strength function. 
To compare a theoretical dipole strength function with our data, we must multiply that strength function by the virtual photon spectrum (Eq. 2) and by our detector response function. Because the angular acceptance of the neutron array is only $5^{\circ}$, the response is a rapidly decreasing function of decay energy. A neutron emitted with $0.3 \mathrm{MeV}$ and at $90^{\circ}$ in the ${ }^{11} \mathrm{Li}$ rest frame, for example, will have an angle of $[0.3 / 30]^{1 / 2}=0.1$, or $6^{\circ}$, in the laboratory; it will miss the neutron array. When the solid curve in the right half of Fig. 5 was Monte-Carloed through our detector response function (and the photon spectrum), it produced the solid curve that fits our data so well in the left half. The solid curve in the right half is the dipole strength function determined by our data. A theoretical model that produces a dipole strength function in agreement with this curve is in agreement with our data.
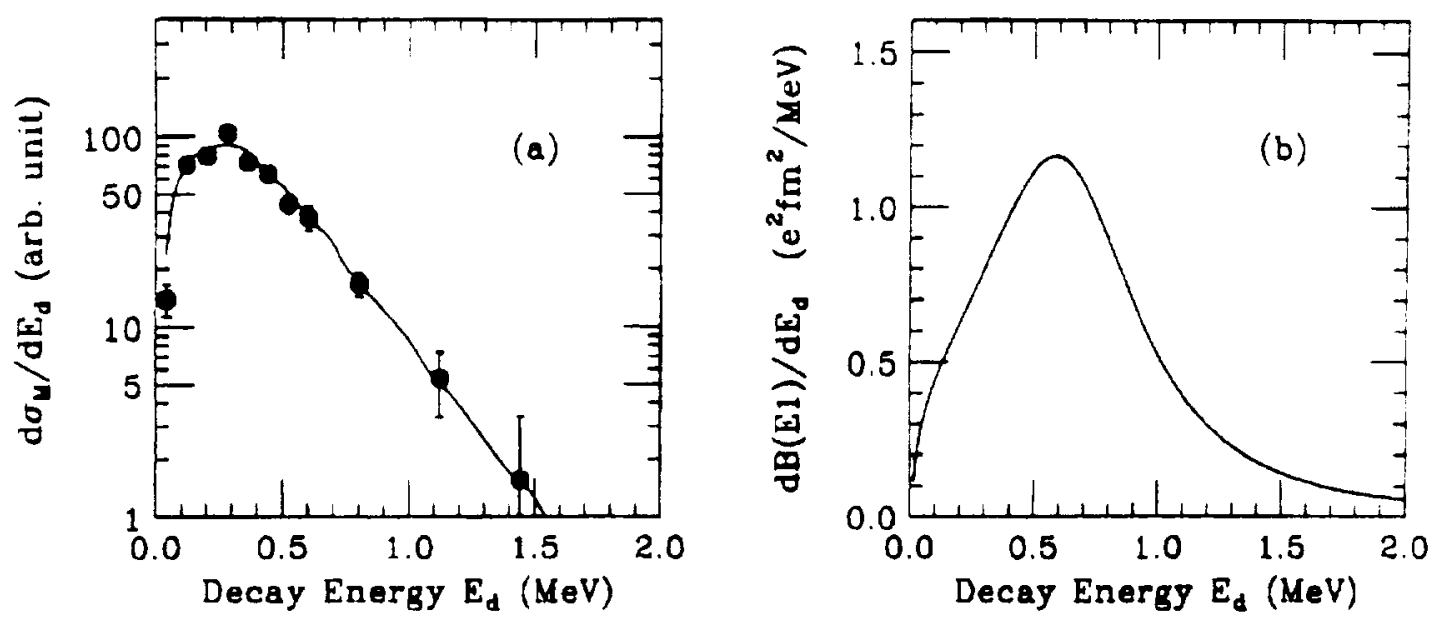

Fig. 5: (a) The measured decay energy spectrum of ${ }^{11} \mathrm{Li}-->{ }^{9} \mathrm{Li}+\mathrm{n}+\mathrm{n}$. (b) The corresponding electric dipole strength function. The solid curve results from a BreitWigner resonance function with $\mathrm{E}_{\mathrm{res}}=0.7 \mathrm{MeV}$ and $\Gamma=0.8 \mathrm{MeV}$. This curve, with the virtual photon spectrum and the detector response function, produced the data-fitting curve in (a).

To obtain the fit that is shown we assumed a Breit-Wigner resonance shape and searched on its two parameters--resonance energy and width. The results were $E_{d}=0.7$ $\mathrm{MeV}\left(\mathrm{E}_{\mathrm{x}}=\mathrm{E}_{\mathrm{d}}+\mathrm{S}_{2 \mathrm{n}} \cong 1.0 \mathrm{MeV}\right)$ and $\Gamma=0.8 \mathrm{MeV}$.

It is instructive to compute the times corresponding to these energies. If our resonance represents the soft dipole resonance, the period of oscillation of the ${ }^{9} \mathrm{Li}$ core in the two-neutron halo may be obtained as follows: 


$$
E_{x}=\hbar \omega=\hbar 2 \pi / T ; T=2 \pi \hbar c / E_{x} c \cong 1250 \mathrm{fm} / \mathrm{c}
$$

From the width of the state we get its lifetime:

$$
\Gamma \tau=\hbar ; \tau=\hbar \mathrm{c} / \Gamma \mathrm{c}=200 / 0.8=250 \mathrm{fm} / \mathrm{c}
$$

Therefore, the state lives for only $1 / 5$ of a oscillation, on average. Such a short lifetime does not support the picture of a core oscillating back and

\subsection{Post-Breakup Coulomb Acceleration}

An observation that was not expected in the experiment is shown in the left half of Fig. 6. The ${ }^{9} \mathrm{Li}$ velocity is systematically higher than the average neutron velocity. To check for a possible instrumental bias we ran a computer simulation of un-accelerated events and got the histogram in the figure. It is symmetrical and centered on zero. Of course, our fragment and neutron velocities were measured by different means, the fragment velocity by the energy deposited in a calibrated CsI scintillator, the neutron velocity by its flight time. Our estimates of the systematic error in each of the two measurements could not account for the observed velocity difference. We were able to
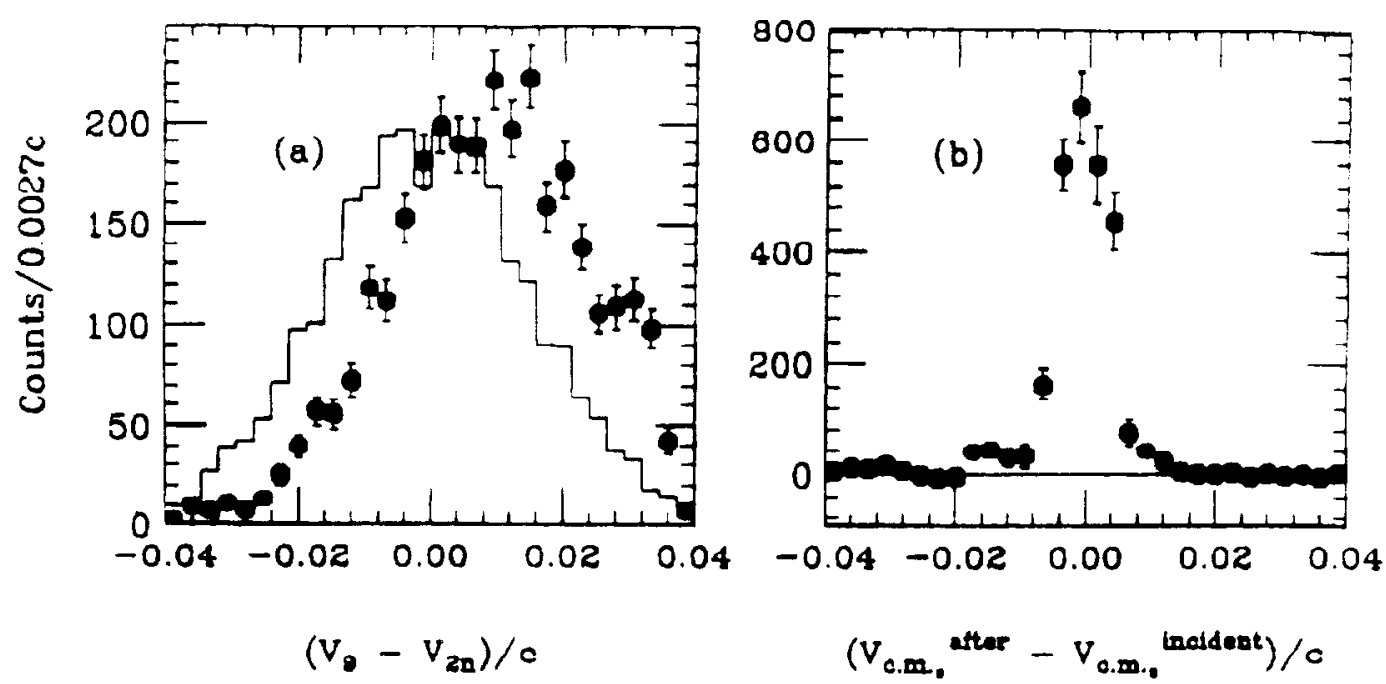

Fig. 6. (a) The spectrum for the velocity difference $V_{9}=V_{2 n}$, where $V_{2 n}$ is the average velocity of the two detected neutrons. The histogram is the result of a Monte-Carlo simulation assuming no Coulomb-acceleration effects. (b) The spectrum for the longitudinal component of the center-of-mass velocity before breakup subtracted from the center-of-mass velocity after breakup. 
construct an experimental check, as illustrated in the right half of Fig. 6. There we compare the initial center-of-mass velocity, ( $\approx$ "Li velocity), with the final $\mathrm{CM}$ velocity. Since the latter value depends mostly on the ${ }^{9} \mathrm{Li}$ velocity, which comes from energy deposition, and the former (and the neutron velocity) comes from time-of-flight measurement, the almost perfect centering of this distribution is an indication that the two methods of determining velocity may not introduce a large systematic error in $v_{9}-v_{2 n}$ In addition, the width of the distribution on the right side gives us a measure of our instrumental velocity-difference resolution.

We have no choice but to take the observed velocity difference seriously and try to interpret it. One possible interpretation is illustrated by the simplified sketch in Fig. 7. This shows that if the lifetime of the excited " $\mathrm{Li}$ is short enough, breakup will occur while the $\mathrm{Li}$ is still high on the Coulomb hill, whereas a long lifetime, such as might result from the excited ${ }^{11} \mathrm{Li}$ going through a resonant state, will result in breakup occuring far from the $\mathrm{Pb}$ target. Only in the former case will the ${ }^{9} \mathrm{Li}$ receive a Coulomb acceleration that the neutrons do not. We can work this idea backwards from the measured value of $V_{9}-V_{2 n}$ and the velocity of the particles to get an estimate of the time between photon absorption and ${ }^{11} \mathrm{Li}$ breakup. The result is $\tau \sim 60 \mathrm{fm} / \mathrm{c}$.

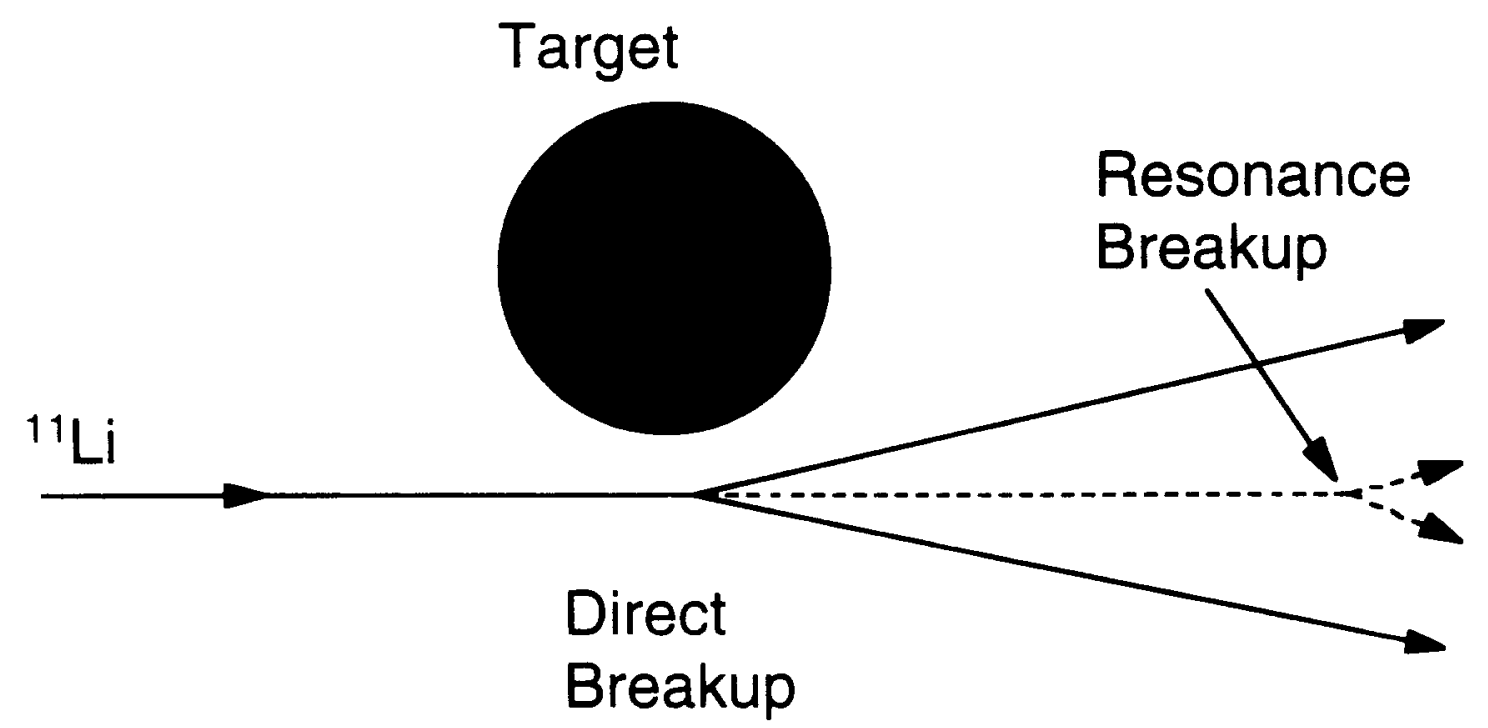

Fig. 7. A schematic view of two possible mechanisms of "Li breakup.

From the width of the "resonance" we deduced a $\tau$ of $250 \mathrm{fm} / \mathrm{c}$. More doubt is cast on the existence of a resonant state. The peak in Fig. 5 looks like a resonance, but an excitation function must rise from zero at threshold and, because of a sum rule, eventually 
come down again. The Coulomb acceleration method of lifetime determination is independent of whether or not there is a soft-dipole resonance, and, of course, $60 \mathrm{fm} / \mathrm{c}$ is only $5 \%$ of the $1250 \mathrm{fm} / \mathrm{c}$ oscillation period for a soft-dipole resonance at $E_{X}=1 \mathrm{MeV}$. There is El strength at low excitation energy, but the system seems not to go into a resonant mode, such as a soft-dipole mode. Rather, we conclude that we have observed the direct photodisintegration of " $\mathrm{Li}$.

\section{THE QUESTION OF A DINEUTRON IN THE GROUND STATE OF ${ }^{11}$ LI}

Destroying the ground state, as we do in photodisintegration, would not seem to be the best way to determine its structure. Perturbing the system so much that it is no longer what it was is reminiscent of the Heisenberg microscope thought experiment. Beyond that fundamental limitation the ground (initial)-state structure can, in principle, be determined from a perfect measurement of the final (continuum) state and an exact knowledge of the transition operator. In practice it can be impossible to fulfill these requirements.

\subsection{How to determine the ground-state structure}

With a direct transition to continuum states, the equivalent photon method could, in fact, be a satisfactory way to look at the ground-state structure of "Li. There are two requirements: 1) The momentum of the absorbed photon should not significantly perturb the motion of the " $\mathrm{Li}$ core or of either neutron, it should be a gentle perturbation, and 2) The photon absorption process should take place so quickly that the positions of the three constituents are not significantly changed, it should be a sudden absorption. If these requirements are met, no theory is required to see the $n$ - $n$ ground-state correlation in the final state.

A look at parts (a) and (b) of Fig. 8 (as in Fig. 16 of Ref. 4) tells us that the first requirement is met, because a typical ${ }^{9} \mathrm{Li}$ momentum is about $30 \mathrm{MeV} / \mathrm{c}$ and a typical neutron momentum is about $18 \mathrm{MeV} / \mathrm{c}$, whereas Fig. 5 gives $\mathrm{p} y \leq 1 \mathrm{MeV} / \mathrm{c}$, which is small in comparison to both $30 \mathrm{MeV} / \mathrm{c}$ and $18 \mathrm{MeV} / \mathrm{c}$.

If our beam velocity had been relativistic, the second requirement would have been met extremely well because the electric field of the $\mathrm{Pb}$ nucleus would have been a flattened pancake as seen by the $\mathrm{Li}$. With an impact parameter of 10-20 fm and a speed of $\mathrm{c}$, the collision time could have been less than $1 \mathrm{fm} / \mathrm{c}$. Photon absorption is sudden if the collision time is much less than the orbital period of the halo neutrons. A halo neutron has. 

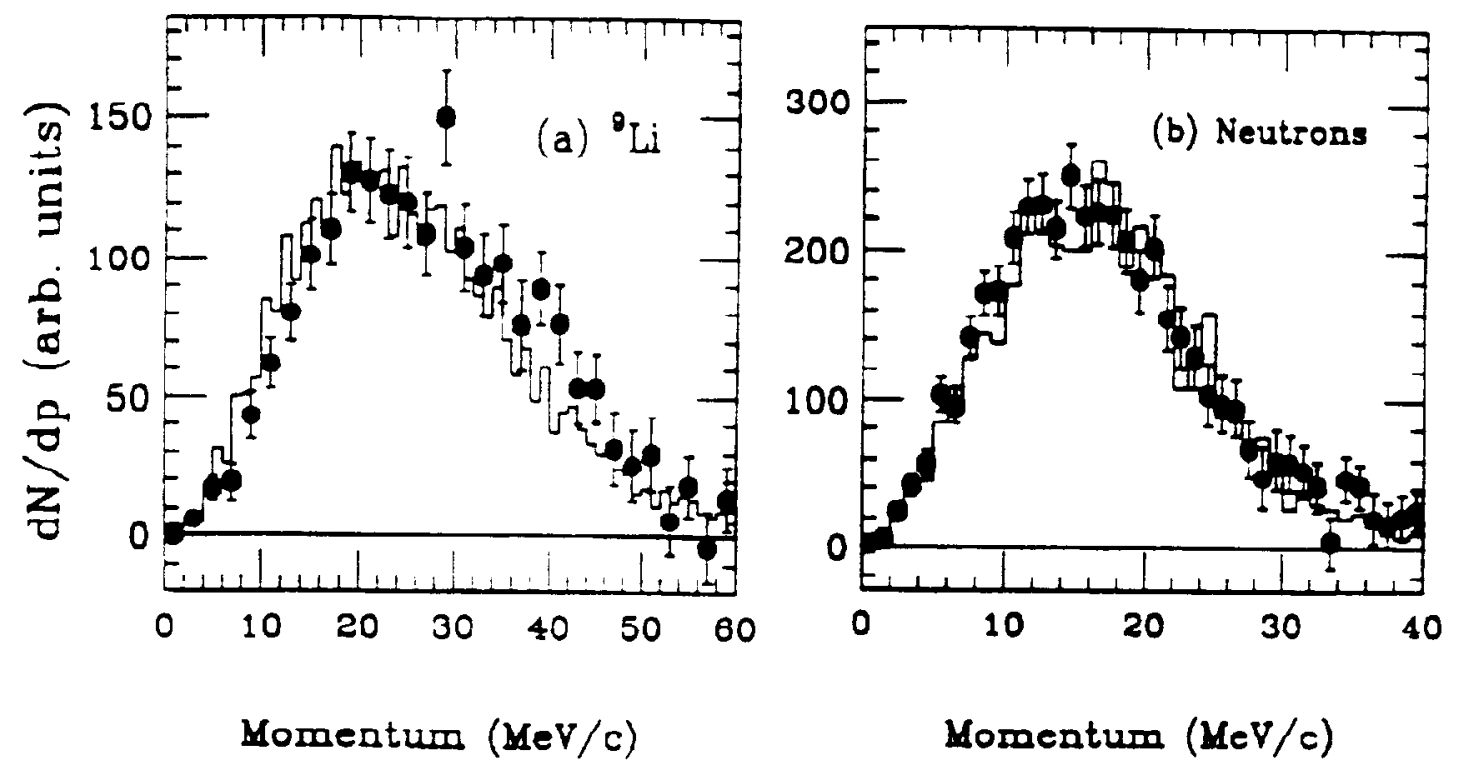

Fig. 8. Momentum spectra of ${ }^{4} \mathrm{Li}$ fragments and of single neutrons. The points are from our experiment, and the histograms are computer simulations, which include them detector response function, of a standard 3-body phase-space breakup of the excited "Li.

a radius $\sim 6 \mathrm{fm}$. With momentum $18 \mathrm{MeV} / \mathrm{c}$, its speed is only $0.02 \mathrm{c}$, and its orbital period $\sim 2,000 \mathrm{fm} / \mathrm{c}$. A collision time of $1 \mathrm{fm} / \mathrm{c}$ would meet the suddenness condition very well At our beam energy of $28 \mathrm{MeV} /$ nucleon $\mathrm{v}=\mathrm{c} / 4$, however, and there is almost no flattenning. Photon absorption occurs over the much longer time of $\sim(25 \mathrm{fm}) /(\mathrm{c} / 4)=100 \mathrm{fm} / \mathrm{c}$, and we saw in Section 3.2 that $\tau \sim 60 \mathrm{fm} / \mathrm{c}$. But even these times are much less than the orbital period. So the second requirement is also satisfied. We can, therefore, expect that a dineutron in the ground state would show itself in the final state. With that expectation, we look for evidence of a dineutron in our data.

\section{2 n - n Angle Distribution}

The best place to look for an $n-n$ correlation is in the distribution function, in the

${ }^{11} \mathrm{Li}$ rest frame, for the angle $\theta$ between the two neutrons. (Partitioning of the "Li decay energy into kinetic energy of the ${ }^{9} \mathrm{Li}-\mathrm{n}^{2}$ system and internal energy of the $n^{2}$ system favored the former. This is evidence for a "modified" dineutron. [5]) If there is no correlation, the distribution function should be independent of $\theta$. The points in Fig. 9 give the observed distribution. It is not isotropic, but the reason turns out to be a consequence of the response of our neutron detector. To illustrate that, we have made a computer simulaton of the $n-n$ angle distribution that would be observed if a standard 3-body phase-space 
distribution governed the $\mathrm{Li}$ decay. The result of that simulation is the histogram that gives a rather good fit to the actual data. The other histogram results from a simulation in which we assume an extreme dineutron correlation, one in which the two neutrons orbit together in ${ }^{11} \mathrm{Li}$ and go off in the same direction with the same velocity upon separation from the ${ }^{9} \mathrm{Li}$ core. The detector response smoothes the prediction but still leaves a strong peak at $\cos \theta=1$. In Fig. 7 that peak goes off scale beyond 2,000--more than ten times the value in our data. Between the two models it is clear that the data favor the phase-space model with no correlation between the neutrons, i.e., no evidence for a dineutron.

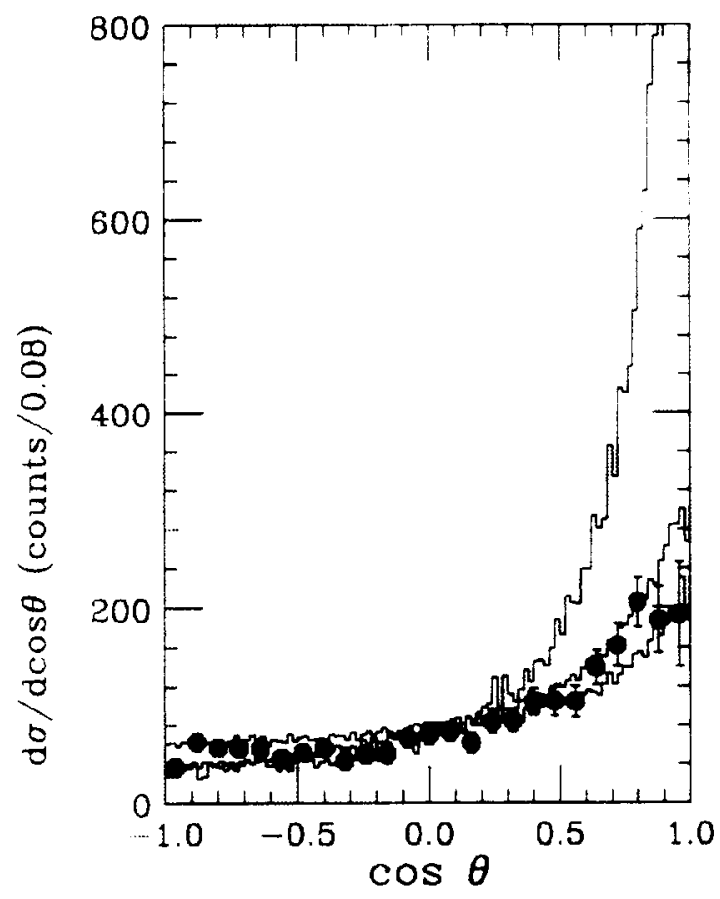

Fig. 9. Angle distribution of the two neutrons when "Li decays. into ${ }^{9} \mathrm{Li}$ $+\mathrm{n}+\mathrm{n}$. The angle between the neutrons is $\theta$. The points are from our experiment. The histograms are Monte-Carlo simulations of two decay models--a standard 3-body phase-space model and an extreme case of a dineutron model. The dineutron histogram rises above 2,000 at $\cos \theta=1$.

\section{IMPROVED NEUTRON DETECTOR--NEUTRON WALLS}

In order to maintain a reasonable energy resolution, the thickness of the neutron detector must be small, resulting in a detection efficiency significantly $<1$, typically $\sim 0.1$. In a two--neutron experiment the efficiency is the 0.01. And with a "beam" of an exotic nucleus the intensity is far below the 1.0 particle $\mu \mathrm{A}\left(6 \times 10^{12 / \mathrm{s}}\right)$ that was once standard in a nuclear physics experiment. Our "Li intensity was $<6 \times 10^{2} / \mathrm{s}$. A large neutron detector, such as the 54-can array in Fig. 2, partially compensates for this deficiency of a factor of $10^{10}$. 
We have built a pair of neutron walls, each consisting of 25 long glass cells filled with NE2 13 liquid scintillator and having phototubes at the ends. This will give us a factor of 10 increase in the volume of scintillator but with only 100 phototubes rather than 540 . The $2 \mathrm{~m} \times 2 \mathrm{~m}$ wall area will give us 3 times the angular acceptance and, therefore, reasonable efficiency for events with 9 times the decay energy. Another advantage of the walls is that the scintillator containment vessels are so thin that scattering of neutrons in them is about one-tenth of wgat it was in the cans and other materials of the arrays. A photograph of one of the walls being assembled by P. Zecher and J.J. Kruse is shown in Fig. 10, and one of the completed walls (without its protective casing) is shown in Fig. 11.

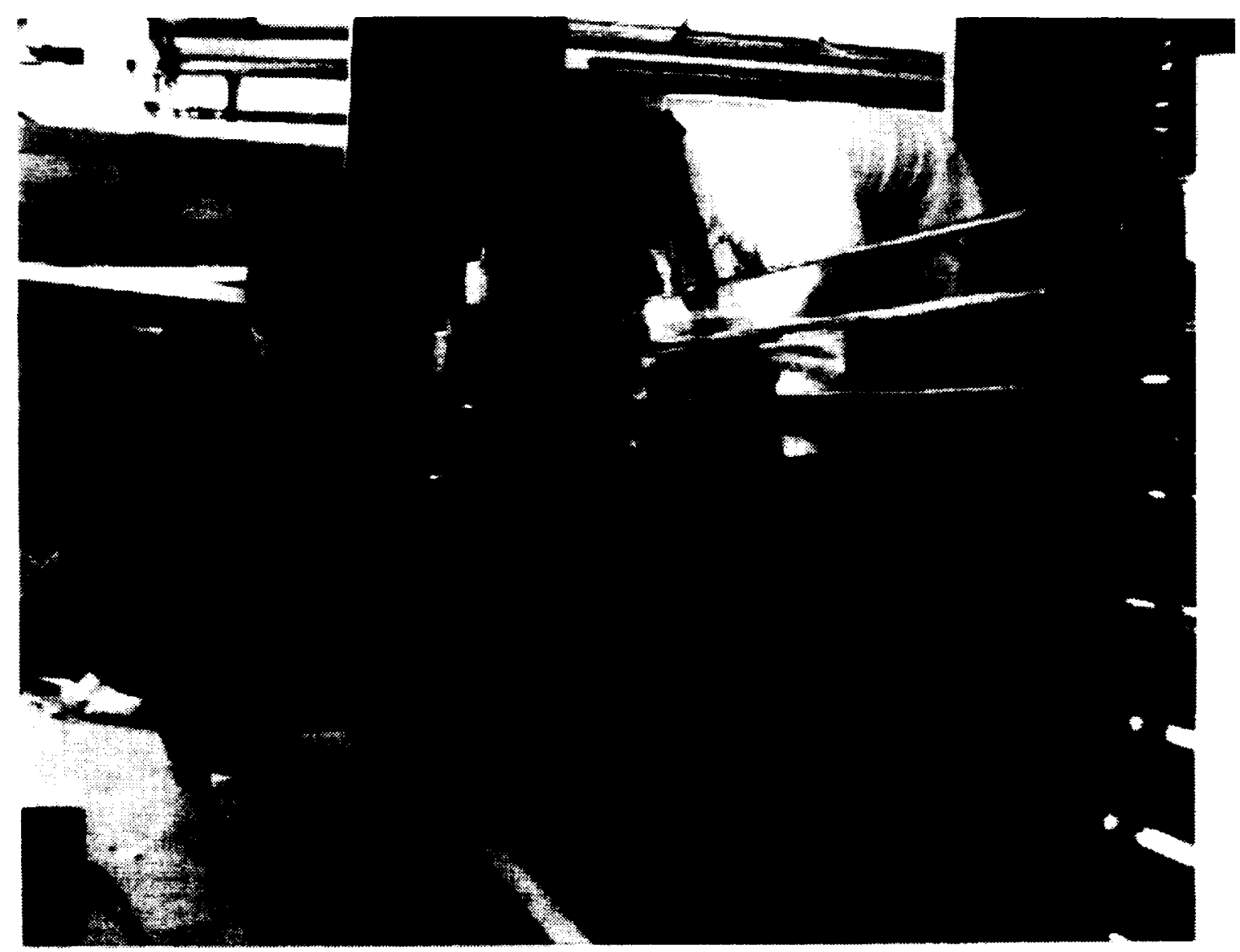

Fig. 10. The first neutron wall being assembled in late March, 1995. 


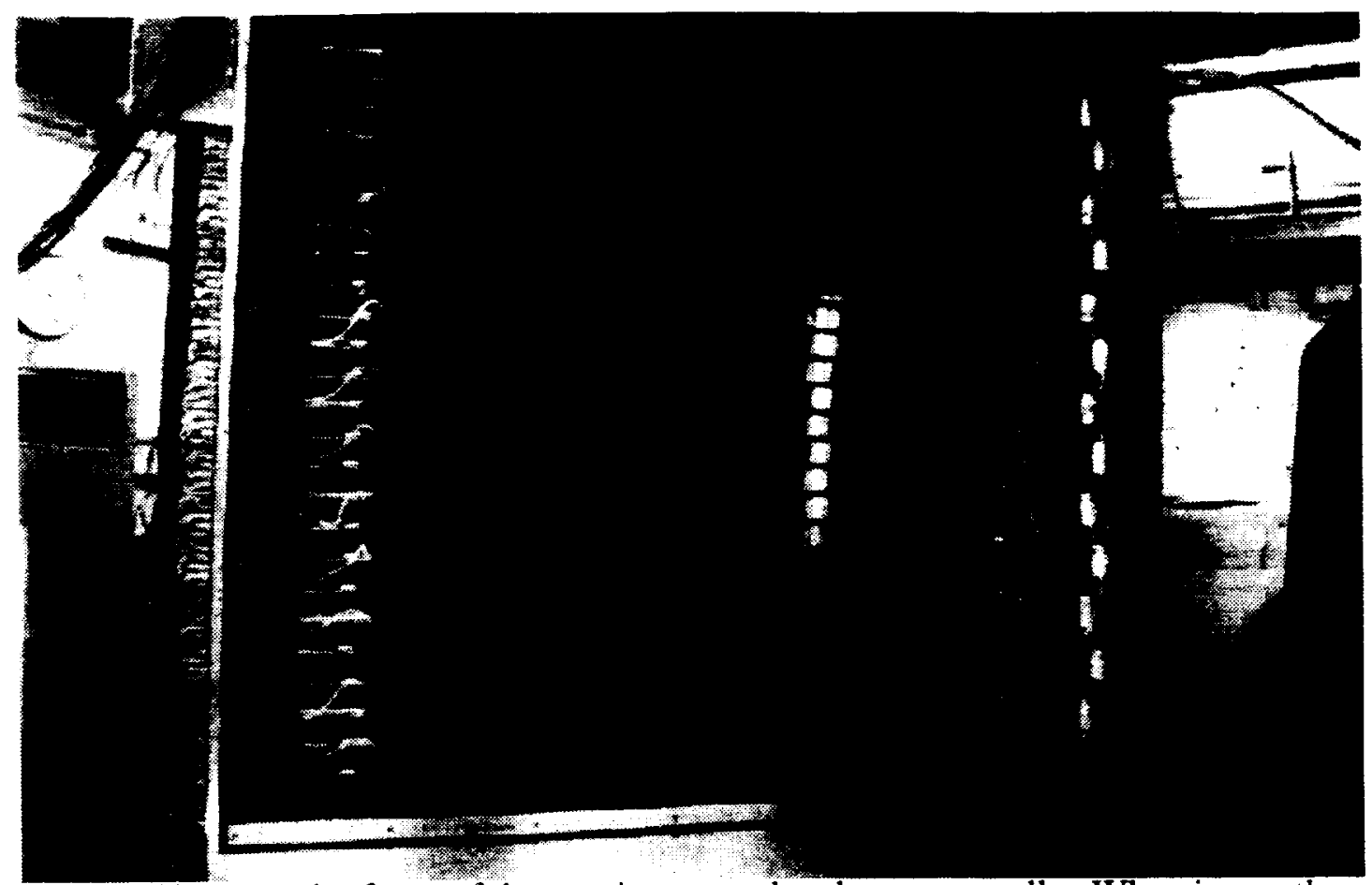

Fig. 11. Photograph of one of the two just-completed neutron walls. When in use there is a cover palte of $1-\mathrm{mm}$ thickness to protect the glass and to keep out light.

\section{Acknowledgements}

We wish to gratefully acknowledge the support of the U.S. National Science

Foundation under Grant Numbers INT91-13997 and PHY92-14992 and of the Hungarian Academy of Sciences.

\section{References}

1. A. B. Migdal, Yad. Fiz. 16 (1972) 427; English translation Sov. J. Nucl. Phys. 16 (1973) 238.

2. P. G. Hansen and B. Jonson, Europhys. Lett. 4 (1987) 409.

3. K. Ieki, D. Sackett, A. Galonsky, C. A. Bertulani, J. J. Kruse, W. G. Lynch, D. J. Morrissey, N. A. Orr, H. Schultz, B. M. Sherrill, A. Sustich, J. A. Winger, F. Deák, Á. Horváth, Á. Kiss, Z. Seres, J. J. Kolata, R. E. Warner and D. L. Humphrey, Phys. Rev. Lett. 70 (1993) 730.

4. E. Sackett, K. Ieki, A. Galonsky, C. A. Bertulani, H. Esbensen, J. J. Kruse, W. G. Lynch, D. J. Morrissey, N. A. Orr, B. M. Sherrill, H. Schulz, A. Sustich, J. A. Winger,F. Deák, A. Horváth, Á. Kiss, Z. Seres, J. J. Kolata, R. E. Warner and D. L. Humphrey, Phys. Rev. C 48 (1993) 118.

5. S. Shimoura, T. Nakamara, M. Ishihara, N. Inabe, T. Kobayashi, T. Kubo, R. H. Siemssen, I. Tanihata and Y. Watanabe, Phys. Lett. B 348 (1995) 29.

6. S.C. Fultz, R.L. Bramblett, J.T. Caldwell and N.A. Kerr, Phys. Rev. 127 (1962) 1273.

7. B. M. Sherrill. D. J. Morrissey. J. A. Nolen and J. A. Winger, Nucl. Instrum. Methods B 56/57 (1991) 1106.

8. G. Baur, C. A. Bertulani and H. Rebel, Nucl. Phys. A458, (1986) 188.

9. C. A. Bertulani and G. Baur, Phys. Rep. 163, (1988) 299.

10. B.M. Young, W. Benenson, M. Fauerbach, J.H. Kelley, R. Pfaff, B.M. Sherrill, M.Steiner, J.S. Winfield. T. Kubo, M. Hellstrom, N.A. Orr, J. Stetson, J.A. Winger and S. Yennello, Phys. Rev. Lett. 71 (1993) 4124. 
